\title{
LIII. On the relative luminous intensities of sun and sky
}

\section{Quirino Majorana}

To cite this article: Quirino Majorana (1901) LIII. On the relative luminous intensities of sun and sky, Philosophical Magazine Series 6, 1:5, 555-562, DOI: 10.1080/14786440109462645

To link to this article: http://dx.doi.org/10.1080/14786440109462645

曲 Published online: 08 Jun 2010.

Submit your article to this journal \lceil

Џ Article views: 4

Q View related articles ¿ 
On the Relative Luminous Intensities of Sun and Sky. 555

in the flash which are present in the emission-spectra, interesting comparisons can be made with photographs of the actual flash-spectrum of the sun. I am also engaged in waking accurate determinations of the dispersion of metallic vapours by means of a metal prism of 45 degrees, furnished with mica windows. The prism is filled with hydrogen, and the metal-say sodium-vaporized in this atmosphere by the application of heat. The results obtained in this way are far superior to those yielded by prismatic flames. The angle of the prism is accurately known, and it is filled with nonluminous sodium vapour of uniform density and under known conditions of temperature and pressure. With this I have obtained much greater curvature of the spectrum in the vicinity of the absorption-lines than that figured by Julius (the total lateral bending in one case being 400 times the distance between the $\mathrm{D}$ lines), and the spectrum is perfectly steady instead of fluttering, as is the case when the deviation is effected by means of a sodium-flame of prismatic form. The work along these lines will be reported in a subsequent paper.

University of Wiseonsin.

LIII. On the Relative Luminous Intensities of Sun and Sky. By Quiriso Majorana*.

TVHE problem regarding the cause and intensity of the light coming from the sky has been dealt, with by numerous writers. Newton was the first to point out that under certain conditions some bodies appeared to be coloured, without, however, possessing any coloration properly so-called.

In the case of the atmosphere, the most probable hypothesis would seem to be that according to which the light coming from the sky is due to the reflexion of sunlight from particles suspended in the atmospuere. Strong support is given to this thenry by the fact that the light coming from the sky is polarized. This, however, does not, according to some, constitute an argument in favour of the view that the coloration of the sky is not a true coloration of the atmosphere.

Whaterer the explanation may be, it is in any case interesting to ascertain the value of the luminous intensity of the sky. This intensity might be expressed in terms of any arbitrary unit; but it is much simpler to refer it to the luminous intensity of the sun, no matter in wbai units the actual

* Translated from the Rendiconti della $R$. Accaciemia dei Lincei, Classe di Scienze fis., mat. e nat., vol. ix. $2 d$ Bem., Ser. $E$, fisc.'3, from a separate impression communicated by the Author.

202 
measurement may have been carried out; since the sun's brightness varies with the season and the time of day, and depends on the amount of atmospheric absorption. It is evident that the variableness in the clearness of the atmosphere affects the two quantities under comparison in the same sense, leaving their ratio unaltered.

The experimental determination of this ratio not only renders unnecessary the use of any artificial source of light, but with proper arrangements furnishes an extremely sensitive method of studying the variations in the luminosity of the sky.

The investigation was first suggested by Lord Kelvin, during his stay at Rome, in April 1899. It presents a difficulty which occurs in almost all photometric determinations-that of having to compare two sources of different colour. For this reason, the measurement is essentially somewhat uncertain. Nevertheless, it is possible by a properly arranged apparatus to study fairly conveniently the grosser variations in the luminosity of the sky.

Measurements more precise than those about to be described have already been carried out by others, but the scope of the presert research is limited to the observation of the influence of the variations in the clearness of the sky on the light emitted by it. Now in order to carry out observations in an atmosphere as free as possible from water-vapour, though somewhat rarefied, it becomes necessary to ascend high mountains, and in that case the simplicity of the observations becomes of paramount importance.

Lord Kelvin advised the construction of an apparatus consisting of two tubes, one of which was to be turned towards the sun and the other towards the sky, each tube having an aperture at one end and a paper screen at the other. By altering the dimensions of the apertures and the lengths of the tubes, it would be possible to adjust matters so as to get equal illumination of the two screens, the observer viewing the latter by covering his head with a black cloth. A knowledge of the lengths of the two tubes and of the diameters of their apertures suffices for the deduction of the ratio of the two luminous intensities-that of the sun, and that of an equal angular area of the sky.

The arrangement proposed by Lord Kelvin, though extremely simple, is not convenient when it is desired to make observations on mountains. Suppose, in fact, that the tube which is to be turned towards the sky is made about $50 \mathrm{~cm}$. long, and has an aperture of abont $5 \mathrm{~cm}$. in diameter. In order to obtain equality of illumination of the two screens, 
it is necessary to make the solar tribe about 1.5 metres long, and its aperture only $\cdot 1$ to $\cdot 2 \mathrm{~mm}$. in diameter. Hence, owing to the inconvenience of making the solar tube very long, there is an uncertainty in the determination of the ratio of the two apertures.

It was therefore preferable to construct a small apparatusa sky-photometer-easily portable, and enabling observations to be taken rapidly. This is represented in fig. 1 . The two

Fig. 1.

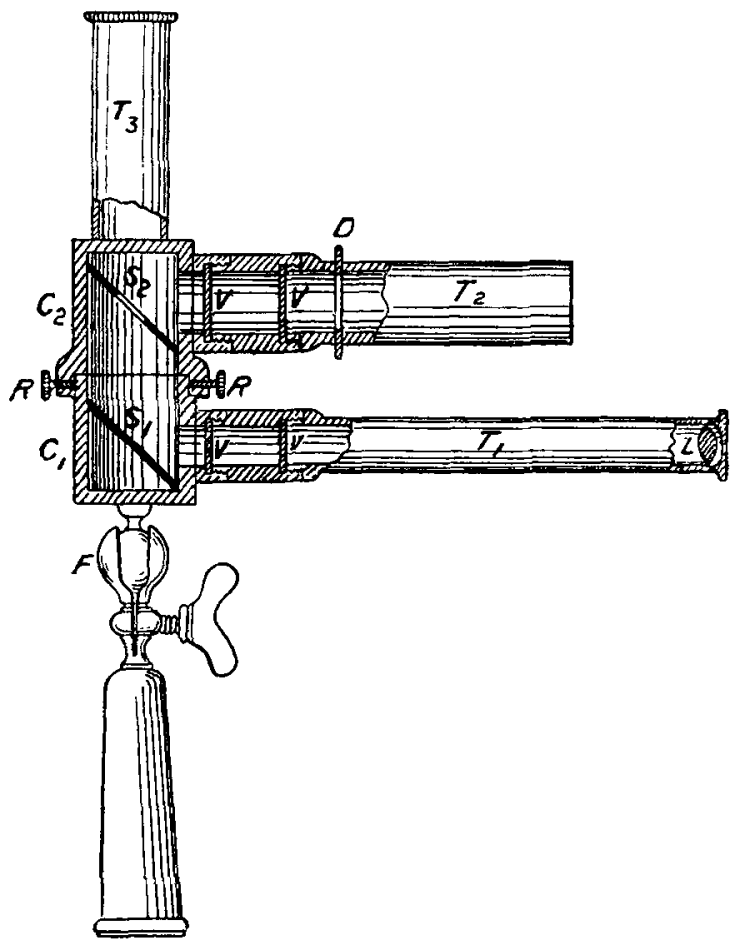

tubes $\mathrm{T}_{1}$ and $\mathrm{T}_{2}$ are intended to be turned towards the sun and sky respectively. They are rigidly fixed to two hollow cylinders, $C_{1}$ and $C_{y}$. These cylinders are coaxial, and $C_{2}$ is capable of rotating respectively to $C_{1}$, and of being fixed in any desired position by means of the screw $R$. The cylinder $\mathrm{C}_{2}$ carries a third tube $\mathrm{T}_{3}$, which serves as the ocular tube. The tube $T_{1}$ is at one end furnished with a small short-focus lens $L$, in front of which is placed a diaphragm with an aperture about $1.5 \mathrm{~mm}$. in diameter. The divergent light coming from the lens passes through two ground-glass screens $V$ in 
succession, and is thereby weakened. By means of this artifice, the illumination of the second ground-glass screen, which is the one observed, is rendered much more uniform. A mirror, $\mathrm{S}_{1}$, reflects the light along the direction of the tube $T_{3}$. The tube $T_{2}$ does not carry a lens, but is provided with a free aperture, and is fitted with an iris diaphragm D, which serves to adjust the intensity of the light falling on the two ground-glass screens similar to those in the tube $T_{1}$. A second mirror, $\mathrm{S}_{2}$, provided with a central aperture, throws the light coming along $\mathrm{T}_{2}$ in the direction of $\mathrm{T}_{3}$. A ball-andsocket joint $\mathrm{F}$ enables the apparatus to be fixed in any position. The instrument therefore allows of the tube $\mathrm{T}_{1}$ being directed towards the sun, and the tube $T_{2}$ towards any other point of the sky. The eye, by means of the ocular tube, thus sees an azure field illuminated by the sky, and in its centre an elliptic spot of a reddish or white colour due to the sun. The aperture of the diaphragm $D$ is adjusted so as to give equal illumination of the two areas.

The instrument described had to be graduated. For each position of the iris diaphragm it was necessary to know the ratio of the sun's luminous intensity to that of an equal angular area of the sky (about $32^{\prime}$ ). The graduation might have been effected by means of a comparison with the tubeinstrument suggested by Kelvin and described at the beginning of this note. But such an operation would have been somewhat uncertain, involving the comparison of lights of different colour. Instead of this, it is better to use a more indirect method, involving the use of artificial sources of light of known intensity, and depending on the following considerations :-

Let (fig. 2) the short-focus lens of the photometer be

Fig. 2.

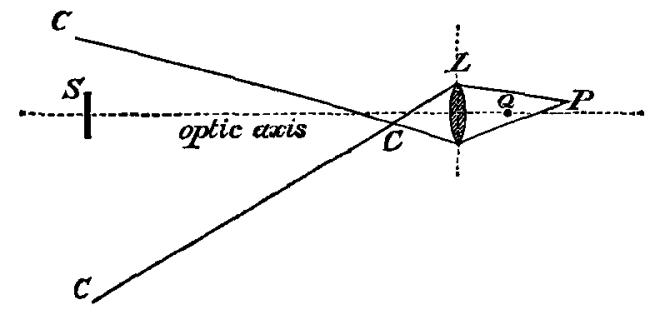

represented by $\mathrm{L}$, and the first ground-glass screen by $\mathrm{S}$. Let the diameters of the lens and the screen $S$ be about equal, and let the distance $L S$ equal several times the focal distance of $L$. Let a source of definite dimensions, representing the 
sun, be placed symmetrically with respect to the optic axis of the system, and at a sufficiently great distance from $\mathrm{L}$. If the dimensions of the source of light be not excessive, every point of it, $\mathrm{P}$, will produce a luminous cone $\mathrm{CCC}$ on the other side of the lens L, of approximately uniform intensity, and within this cone will be comprised the screen S. Provided the luminous cone is uniform, each point of the source will contribute the same amount towards the illumination of $\mathrm{S}$. Thus the amount of light falling on $\mathrm{S}$ is always the same, whether the source of light be of finite dimensions, or a point-source. It is evident that in order that all this may hold, certain conditions must be satisfied. It is, in fact, necessary that the focal length of the lens $L$ should be short in comparison with the length of LS ; and that the luminous source should, besides being of sufficiently small dimensions, be at a sufficiently great distance from the external focus $Q$. A preliminary test of the apparatus is sufficient to show whether these conditions are fulfilled in every respect. If they are, then it is possible to regard the dimensions of the luminous source $P$ as arbitrary, and to suppose, for instance, that it subtends the same angle of $32^{\prime}$ as the sun. For the sky is substituted a circular uniformly luminous screen, of intensity $i$. Let this sereen be sufficiently large in comparison with its distance from the first ground-glass screen in the tube T (see fig. 3).

Fig. 3.

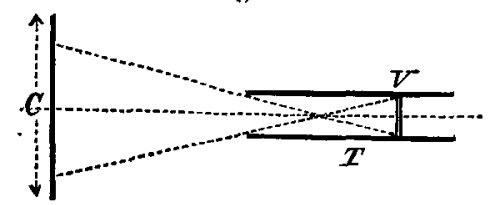

It is next necessary to vary the intensities of the two sources in order to calibrate the instrument. But since we are concerned merely with the ratio of their intensities, it is evidently sufficient to vary one of them only. The screen representing the sky may be allowed to remain in a fixed position, while the distance of the source representing the sun is varied.

In doing this we may still suppose, as explained above, that the source subtends an angle of $32^{\prime}$; while its intensity depends on its real distance from $\mathrm{L}$.

In order to graduate the photometer, it is necessary to determine the ratio of the intensities of the two sources, and for this purpose an ordinary photometer may be used. If I stand for the intensity of the source representing the sun, and 
$i$ for that of the screen which is a partial representation of the sky, then by means of such a photometer we find the value of

$$
\mathrm{K}=\frac{I}{i} \text {. }
$$

Let now our sky photometer be introduced between the sereen $\mathrm{C}$ and the source $\mathrm{S}$ (fig. 4), care being taken, by means

Fig. 4.

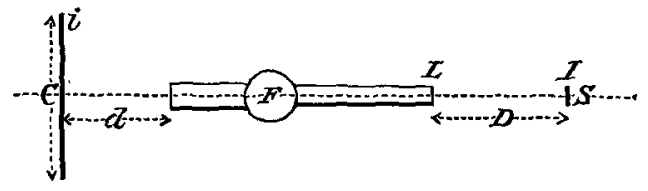

of suitable arrangements, to prevent the light from $S$ reaching C. Further, $C$ is placed sufficiently near the photometer, for the reason already stated. Using the ocular tube, we adjust the iris diaphragm until equality of illumination is obtained. The reading $n$ on the scale of the iris diaphragm is then noted. The problem is to find the value of $r$ corresponding to the reading $n$. Now if $\alpha$ stand for the visual angle of the sun, expressed in minutes, and $d$ for the distance of the screen $C$ from the photometer, then

$$
m=\left(\frac{216000}{2 \pi d a}\right)^{2}
$$

is the number of times which the area of C contains a circular area subtending an angle $a$ at a distance $d$.

If $D$ is the distance of the source 1 from the photometer. the quantity

$$
\mathrm{K} \frac{d^{2}}{\mathrm{D}^{2}}
$$

is $m$ times greater than the required ratio. Hence to the graduation $n$ of the diaphragm corresponds the value

$$
r=\mathrm{K} \frac{d^{2}}{\mathrm{D}^{2}}\left(\frac{21600 \mathrm{C}}{2 \pi d a}\right)^{2}=\mathrm{K}\left(\frac{21600 \mathrm{C}}{2 \pi \alpha \mathrm{D}}\right)^{2}
$$

As will be seen, this value is independent of the distance of the screen $\mathrm{C}$ from the photometer; as should be the case if the condition indicated by fig. 3 is satisfied.

To each value of $\mathbf{D}$ corresponds a pair of values of $n$ and $r$; these values may be used for constructing a calibration table.

The operation of calibrating the photometer presents no difficulty, since the two sources $C$ and $S$.may be chosen so as 
to be of the same tint. But the matter is somewhat more uncertain when the comparison is to be made between the light of the sun and that of the sky ; especially if the difference of colour is strongly marked, as at the crater of Etna, some 3000 metres above sea-level. In such a case it is necessary to repeat the measurement a large number of times, re-setting the diaphragm each time.

With this apparatus, measurements were carried out at Rome in the summer of last year; they were repeated, with greater frequency and regularity, at Catania during the same period. The following are some of the results obtained.

The luminosity of the sky varies from point to point.

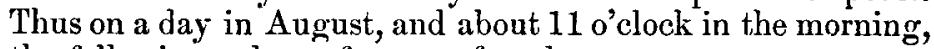
the following values of $r$ were found :-

In the immediate neighbourhood of the sun $. r=357,000$

About $90^{\circ}$ from the sun, in any direction . $r=950,000$

Almost on the horizon . . . . . . $r=98,000$

A white cloud . . . . . . . . $r=18,000$

These variations are evidently due for the most part to the presence of aqueous vapour in the atmosphere.

But the luminosity of the sky also varies throughout the day. In order to ascertain this, observations extending over a period of seven hours were made on the 5th of August, 1899 . The two tubes of the sky-photometer were placed at an angle of $90^{\circ}$ relatively to each other. The first tube having been directed towards the sun, the second was moved towards the least luminous portion of the sky. The following numbers are the means of the observations made on that day :-

Hours after Midnight.

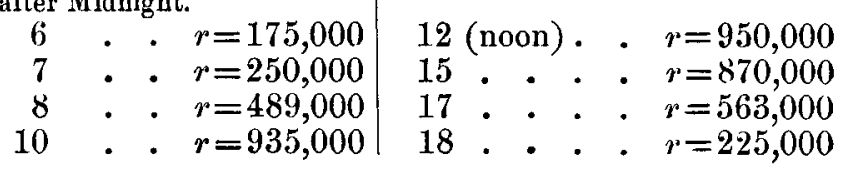

The table shows that the relative luminosity of the sky is least during the hottest part of the day, and greatest at sunrise and sunset.

But the figures given above change considerably with the altitude. In order to study this point, observations were made at the Etna Observatory*, from the 8th to the 13th August, 1899. It was in the first place easy to ascertain the

* The Etna Observatory is a little below the level of the crater. It is 2942 metres above sea-level, and its latitude is $37^{\circ} 44^{\prime} 17^{\prime \prime}$. I am indebted to Prof. A. Riccò for the hospitality offered to me, which enabled me to make these observations. 


\section{On the Relative Luminous Intensities of Sun and Sky.}

fact that-except for regions close to the horizon-the luminosity of the sky had a sensibly constant value in all directions when viewed from the summit of Etna.

Further, points in the neighbourhood of the sun gave the same value of $r$ as those in any other region*.

It was also found at this altitude that the value of $r$ increases from morning to mid-day. Thus, on the 11 th of August, 1899, when the morning was an exceptionally clear one, the following observations were obtained :-

$\begin{array}{cc}\text { Time. } & r . \\ 5 \cdot 50 & 2,570,000 \\ 7 & 3,125,000 \\ 8 & 3,650,000 \\ 9 & 3,930,000 \\ 11 & 3,760,000\end{array}$

Thus the value of $r$ at the crater of Etna is about five times greater than in Catania.

Almost simultaneously with these observations, Sig. Gaudenzio Sella carried out a similar series on Monte Rosa. He used the photometer described above, consisting of plain cardboard tubes, thus realising the apparatus suggested by Lord Kelvin. He found values of $r$ oscillating about 5,000,000, and thus not differing very greatly from those found for Etna.

Considering the probable connexion between the quantity of aqueous vapour in the atmosphere, in the form of a light mist, and the luminosity of the sky, an attempt was made to ascertain whether the hygrometric state of the place of observation had any influence on the clearness of the sky. With this object in view, almost all the observations made were accompanied by determinations of the absolute and relative humidity, by means of a good wet-and-dry bulb hygrometer. No relation, however, was found, probably because the hygrometric state of the lower atmospheric strata may be quite independent of that of the upper ones, to which the colour of the sky is due.

* It is, however, to be noted that it is impossible to push the obserrations to points in the immediate neighbourhood of the sun, since there is then the danger that the light coming from the sun may enter the skytube. 